Article

\title{
Hurwitz-Lerch Type Multi-Poly-Cauchy Numbers
}

\author{
Noel Lacpao ${ }^{1, * \mathbb{D}}$, Roberto Corcino ${ }^{2} \mathbb{D}$ and Mary Ann Ritzell Vega ${ }^{3}$
}

1 Department of Mathematics, College of Arts and Sciences, Bukidnon State University, Malaybalay City 8700, Philippines

2 Research Institute for Computational Mathematics and Physics, Cebu Normal University, Cebu City 6000, Philippines; rcorcino@yahoo.com

3 Department of Mathematics and Statistics, Mindanao State University-Iligan Institute of Technology, Iligan City 9200, Philippines; maryannritzell.vega@g.msuiit.edu.ph

* Correspondence: noel.lacpao@g.msuiit.edu.ph; Tel.: +63-998-9896959

Received: 24 January 2019; Accepted: 4 April 2019; Published: 7 April 2019

stacker tor updates

Abstract: In this paper, we define Hurwitz-Lerch multi-poly-Cauchy numbers using the multiple polylogarithm factorial function. Furthermore, we establish properties of these types of numbers and obtain two different forms of the explicit formula using Stirling numbers of the first kind.

Keywords: multiple polylogarithm functions; poly-Cauchy numbers of the first and second kind; Hurwitz-Lerch factorial zeta function; generating function

MSC: Primary: 11M35; Secondary: 11B68; 11B73; 11B75; 11G55

\section{Introduction}

The Cauchy numbers [1-3] of the first and second kind, respectively denoted by $c_{n}$ and $\hat{c}_{n}$, play important roles in many applications in number theory, combinatorics, and in different areas such as approximate integrals and difference-differential equations. These sequences of numbers are usually defined by means of the integral,

$$
c_{n}=\int_{0}^{1} t(t-1) \cdots(t-n+1) d t=n ! \int_{0}^{1}\left(\begin{array}{l}
t \\
n
\end{array}\right) d t
$$

and:

$$
\hat{c}_{n}=\int_{0}^{1}(-t)(-t-1) \cdots(-t-n+1) d t=(-1)^{n} \int_{0}^{1}\langle x\rangle_{n} d t
$$

where $\langle x\rangle_{n}=(x)(x+1) \cdots(x+n-1)(n \geq 1)$ is the rising factorial with $\langle x\rangle_{0}=1$.

Bernoulli numbers are defined by the generating function:

$$
\frac{t}{e^{t}-1}=\sum_{n=0}^{\infty} B_{n} \frac{t^{n}}{n !}
$$

One of the relations of the Bernoulli numbers with Stirling numbers of the second kind is:

$$
B_{n}=(-1)^{n} \sum_{m=0}^{n}\left\{\begin{array}{l}
n \\
m
\end{array}\right\} \frac{(-1)^{m} m !}{m+1}
$$

where $\left\{\begin{array}{c}n \\ m\end{array}\right\}$ represents the Stirling numbers of the second kind [4] defined by:

$$
\frac{\left(e^{t}-1\right)^{m}}{m !}=\sum_{n=m}^{\infty}\left\{\begin{array}{c}
n \\
m
\end{array}\right\} \frac{t^{n}}{n !} .
$$


In 1997, Kaneko [5] defined certain variations of Bernoulli numbers in terms of the polylogarithm function:

$$
\operatorname{Li}_{k}(z)=\sum_{n=1}^{\infty} \frac{z^{n}}{n^{k}}
$$

which are called poly-Bernoulli numbers, denoted by $B_{n}^{(k)}$. More precisely, poly-Bernoulli numbers are defined by:

$$
\frac{\mathrm{Li}_{k}\left(1-e^{-t}\right)}{1-e^{-t}}=\sum_{n=0}^{\infty} B_{n}^{(k)} \frac{t^{n}}{n !} .
$$

Parallel to this, certain variations of Cauchy numbers of the first kind were introduced by Komatsu [2,6], whose motivation was to relate the numbers to polylogarithm factorial functions:

$$
\operatorname{Lif}_{k}(z)=\sum_{m=0}^{\infty} \frac{z^{m}}{m !(m+1)^{k}}
$$

These numbers are called poly-Cauchy numbers of the first kind, denoted by $c_{n}^{(k)}$. More precisely, these numbers are defined as follows:

$$
c_{n}^{(k)}=n ! \int_{0}^{1} \cdots \int_{0}^{1}\left(\begin{array}{c}
x_{1} x_{2} \cdots x_{k} \\
n
\end{array}\right) d x_{1} d x_{2} \cdots d x_{k} .
$$

The poly-Cauchy numbers possess several properties including an explicit formula:

$$
c_{n}^{(k)}=(-1)^{n} \sum_{m=0}^{n}\left[\begin{array}{c}
n \\
m
\end{array}\right] \frac{(-1)^{m}}{(m+1)^{k}}
$$

where $\left[\begin{array}{l}n \\ m\end{array}\right]$ represents the Stirling numbers of the first kind [4] defined by:

$$
\frac{\left[\log (1+t)^{m}\right]}{m !}=\sum_{n=m}^{\infty}(-1)^{n-m}\left[\begin{array}{c}
n \\
m
\end{array}\right] \frac{t^{n}}{n !},
$$

the generating function:

$$
\operatorname{Lif}_{k}(\ln (1+t))=\sum_{n=0}^{\infty} c_{n}^{(k)} \frac{t^{n}}{n !}
$$

and the following relation with Stirling numbers of the second kind:

$$
\sum_{m=0}^{n}\left\{\begin{array}{c}
n \\
m
\end{array}\right\} c_{m}^{(k)}=\frac{1}{(n+1)^{k}}
$$

Komatsu [2,6] also defined poly-Cauchy numbers of the second kind as follows:

$$
\hat{c}_{n}^{(k)}=n ! \int_{0}^{1} \cdots \int_{0}^{1}\left(\begin{array}{c}
-x_{1} x_{2} \cdots x_{k} \\
n
\end{array}\right) d x_{1} d x_{2} \cdots d x_{k} .
$$

Clearly, $\hat{c}_{n}=\hat{c}_{n}^{(1)}$. Similarly, these numbers possess the following properties: the explicit formula:

$$
\hat{c}_{n}^{(k)}=(-1)^{n} \sum_{m=0}^{n}\left[\begin{array}{l}
n \\
m
\end{array}\right] \frac{1}{(m+1)^{k}}
$$

the generating function:

$$
\operatorname{Lif}_{k}(-\ln (1+t))=\sum_{n=0}^{\infty} \hat{c}_{n}^{(k)} \frac{t^{n}}{n !}
$$


and a relation with Stirling numbers of the second kind:

$$
\sum_{m=0}^{n}\left\{\begin{array}{c}
n \\
m
\end{array}\right\} \hat{c}_{m}^{(k)}=\frac{(-1)^{n}}{(n+1)^{k}}
$$

Certain generalizations of poly-Cauchy numbers of the first and second kind were introduced by Cenkci and Young [7]. This generalization was motivated by the concept of the Hurwitz-Lerch factorial zeta function defined by:

$$
\Phi f(z, s, a)=\sum_{n=0}^{\infty} \frac{z^{n}}{n !(n+a)^{s}}
$$

for $s \in \mathbb{C}$ when $|z|<1$, Res $>1$ when $|z|=1$ and $a \notin\{0,-1,-2, \cdots\}$.

These numbers were called Hurwitz-type poly-Cauchy numbers of the first and second kind, denoted by $c_{n}^{(k)}(a)$ and $\hat{c}_{n}^{(k)}(a)$, which are respectively defined by:

$$
\Phi f(\log (1+t), k, a)=\sum_{n=0}^{\infty} c_{n}^{(k)}(a) \frac{t^{n}}{n !}
$$

and:

$$
\Phi f(-\log (1+t), k, a)=\sum_{n=0}^{\infty} \hat{c}_{n}^{(k)}(a) \frac{t^{n}}{n !} .
$$

These numbers possess the following properties, which are parallel to those of poly-Cauchy numbers: the explicit formulas:

$$
\begin{aligned}
& c_{n}^{(k)}(a)=(-1)^{n} \sum_{m=0}^{n} \frac{(-1)^{m}\left[\begin{array}{l}
n \\
m
\end{array}\right]}{(m+a)^{k}} \\
& \hat{c}_{n}^{(k)}(a)=(-1)^{n} \sum_{m=0}^{n} \frac{\left[\begin{array}{l}
n \\
m
\end{array}\right]}{(m+a)^{k}}
\end{aligned}
$$

relations with Stirling numbers of the second kind:

$$
\begin{aligned}
& \sum_{m=0}^{n}\left\{\begin{array}{l}
n \\
m
\end{array}\right\} c_{m}^{(k)}(a)=\frac{1}{(n+a)^{k}}, \\
& \sum_{m=0}^{n}\left\{\begin{array}{l}
n \\
m
\end{array}\right\} \hat{c}_{m}^{(k)}(a)=\frac{(-1)^{n}}{(n+a)^{k}},
\end{aligned}
$$

and expressions of Hurwitz-type poly-Bernoulli numbers in terms of Hurwitz-type poly-Cauchy numbers:

$$
\begin{aligned}
& B_{n}^{(k)}(a)=\sum_{l=0}^{n} \sum_{m=0}^{n}(-1)^{m+n} m !\left\{\begin{array}{c}
n \\
m
\end{array}\right\}\left\{\begin{array}{c}
m \\
l
\end{array}\right\} c_{l}^{(k)}(a), \\
& B_{n}^{(k)}(a)=\sum_{l=0}^{n} \sum_{m=0}^{n}(-1)^{m} m !\left\{\begin{array}{c}
n \\
m
\end{array}\right\}\left\{\begin{array}{c}
m \\
l
\end{array}\right\} \hat{c}_{l}^{(k)}(a), \\
& c_{n}^{(k)}(a)=\sum_{l=0}^{n} \sum_{m=0}^{n} \frac{(-1)^{m+n}}{m !}\left[\begin{array}{c}
n \\
m
\end{array}\right]\left[\begin{array}{c}
m \\
l
\end{array}\right] B_{l}^{(k)}(a), \\
& \hat{c}_{n}^{(k)}(a)=\sum_{l=0}^{n} \sum_{m=0}^{n} \frac{(-1)^{n}}{m !}\left[\begin{array}{c}
n \\
m
\end{array}\right]\left[\begin{array}{c}
m \\
l
\end{array}\right] B_{l}^{(k)}(a) .
\end{aligned}
$$


Corcino et al. [8,9] extended the poly-Bernoulli numbers and defined the Hurwitz-Lerch-type multi-poly-Bernoulli numbers $B_{n}^{\left(k_{1}, k_{2}, \cdots, k_{r}\right)}(a)$ as follows:

$$
\Phi\left(1-e^{-t},\left(k_{1}, k_{2}, \cdots, k_{r}\right) \cdot a\right)=\sum_{n=0}^{\infty} B_{n}^{\left(k_{1}, k_{2}, \cdots, k_{r}\right)}(a) \frac{t^{n}}{n !} .
$$

The numbers have the explicit formula:

$$
B_{n}^{\left(k_{1}, k_{2}, \cdots, k_{r}\right)}(a)=\sum_{0 \leq m_{1} \leq m_{2} \leq \cdots \leq m_{r} \leq n} \frac{(-1)^{n+m_{r}} m_{r} !\left\{\begin{array}{c}
n \\
m_{r}
\end{array}\right\}}{\left(m_{1}+a-r+1\right)^{k_{1}\left(m_{2}+a-r+2\right)^{k_{2}} \cdots\left(m_{r}+a\right)^{k_{r}}}} .
$$

Further generalization of poly-Cauchy numbers of the first and second kind was defined by Komatsu et al. [10] using the multiple polylogarithm factorial function inspired by the following definition of multi-poly-Bernoulli numbers by Imatomi et al. [11],

$$
\frac{\mathrm{Li}_{k_{1}, k_{2}, \cdots, k_{r}}\left(1-e^{-t}\right)}{1-e^{-t}}=\sum_{n=0}^{\infty} B_{n}^{\left(k_{1}, k_{2}, \cdots, k_{r}\right)} \frac{t^{n}}{n !}
$$

where:

$$
\operatorname{Li}_{k_{1}, k_{2}, \cdots, k_{r}}(z)=\sum_{0 \leq m_{1}<m_{2}<\cdots<m_{r}} \frac{z^{m_{r}}}{m_{1}^{k_{1}} \cdots m_{r}^{k_{r}}}
$$

is the multiple polylogarithm function. Komatsu et al. correspondingly defined a factorial version of the multiple polylogarithm factorial function as follows:

$$
\operatorname{Lif}_{k_{1}, k_{2}, \cdots, k_{r}}(z)=\sum_{0 \leq m_{1}<m_{2}<\cdots<m_{r}} \frac{z^{m_{r}}}{m_{1} ! \cdots m_{r} !\left(m_{1}+1\right)^{k_{1}} \cdots\left(m_{r}+1\right)^{k_{r}}} .
$$

More precisely, the multi-poly-Cauchy numbers of the first and second kinds, $c_{n}^{\left(k_{1}, k_{2}, \cdots, k_{r}\right)}$ and $\hat{c}_{n}^{\left(k_{1}, k_{2}, \cdots, k_{r}\right)}$, are defined, respectively, as:

$$
\begin{aligned}
\operatorname{Lif}_{k_{1}, k_{2}, \cdots, k_{r}}(\ln (1+t)) & =\sum_{n=0}^{\infty} c_{n}^{\left(k_{1}, k_{2}, \cdots, k_{r}\right)} \frac{t^{n}}{n !}, \\
\operatorname{Lif}_{k_{1}, k_{2}, \cdots, k_{r}}(-\ln (1+t)) & =\sum_{n=0}^{\infty} \hat{c}_{n}^{\left(k_{1}, k_{2}, \cdots, k_{r}\right)} \frac{t^{n}}{n !} .
\end{aligned}
$$

These numbers possess the following explicit formula:

$$
\begin{aligned}
& c_{n}^{\left(k_{1}, k_{2}, \cdots, k_{r}\right)}=\sum_{0 \leq m_{1}<m_{2}<\cdots<m_{r} \leq n} \frac{\left[\begin{array}{c}
n \\
m_{r}
\end{array}\right]}{\hat{c}_{n} ! \cdots m_{r-1} !\left(m_{1}+1\right)^{k_{1}} \cdots\left(m_{r}+1\right)^{k_{r}}}, \\
& \hat{c}_{n}^{\left(k_{1}, k_{2}, \cdots, k_{r}\right)}=\sum_{0 \leq m_{1}<m_{2}<\cdots<m_{r} \leq n} \frac{(-1)^{m_{r}}\left[\begin{array}{c}
n \\
m_{r}
\end{array}\right]}{m_{1} ! \cdots m_{r-1} !\left(m_{1}+1\right)^{k_{1}} \cdots\left(m_{r}+1\right)^{k_{r}}} .
\end{aligned}
$$

In this paper, a multiple parameter Hurwitz-Lerch factorial zeta function will be introduced, which will be used to define certain multiple parameter Hurwitz-Lerch poly-Cauchy numbers. Several properties are established including generating functions, explicit formulas, and some relations involving the Stirling numbers.

\section{Hurwitz-Lerch Multi-Poly-Cauchy Numbers}

Combining the concepts of the Hurwitz-Lerch multi-poly-zeta function and multiple polylogarithm factorial function, we can define the Hurwitz-Lerch multi-factorial zeta function as follows: 
Definition 1. The Hurwitz-Lerch multi-factorial zeta function $\Phi f\left(z,\left(k_{1}, k_{2}, \cdots, k_{r}\right)\right.$, a) is defined by:

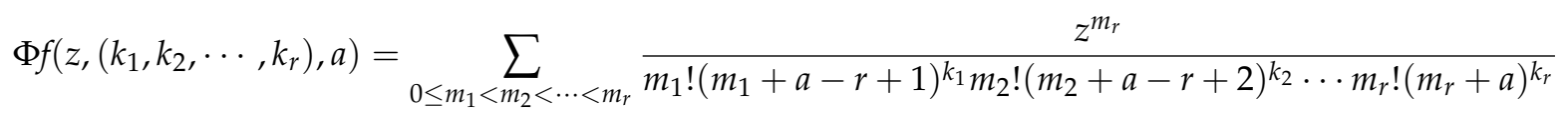
where $k_{i} \in \mathbb{C}, i \in \mathbb{N}$ when $|z|<1$ and $\operatorname{Re} k_{i}>1$ when $|z|=1$ and $a \notin\{0,-1,-2, \cdots\}$.

Note that when $r=1$, Definition 1 gives:

$$
\Phi f\left(z, k_{1}, a\right)=\sum_{0 \leq m_{1}} \frac{z^{m_{1}}}{m_{1} !\left(m_{1}+a\right)^{k_{1}}}
$$

the Hurwitz-Lerch factorial zeta function.

Observe that:

$$
\begin{aligned}
& \operatorname{\Phi f}\left(\ln (1+t),\left(k_{1}, k_{2}, \cdots, k_{r}\right), a\right) \\
= & \sum_{0 \leq m_{1}<m_{2}<\cdots<m_{r}} \frac{(\ln (1+t))^{m_{r}}}{m_{1} !\left(m_{1}+a-r+1\right)^{k_{1}} m_{2} !\left(m_{2}+a-r+2\right)^{k_{2}} \cdots m_{r} !\left(m_{r}+a\right)^{k_{r}}} \\
= & \operatorname{Lif}_{k_{1}, k_{2}, \cdots, k_{r}}(\ln (1+t))(a) .
\end{aligned}
$$

Comparing this with the left-hand side of (5) and (6), it would be logical to define the Hurwitz-Lerch multi-poly-Cauchy numbers of the first and second kinds as follows:

Definition 2. The Hurwitz-Lerch-type multi-poly-Cauchy numbers of the first kind are defined by:

$$
\Phi f\left(\ln (1+t),\left(k_{1}, k_{2}, \cdots, k_{r}\right), a\right)=\sum_{n=0}^{\infty} c_{n}^{\left(k_{1}, k_{2}, \cdots, k_{r}\right)}(a) \frac{t^{n}}{n !}
$$

Definition 3. The Hurwitz-Lerch-type multi-poly-Cauchy numbers of the second kind are defined by:

$$
\Phi f\left(-\ln (1+t),\left(k_{1}, k_{2}, \cdots, k_{r}\right), a\right)=\sum_{n=0}^{\infty} \hat{c}_{n}^{\left(k_{1}, k_{2}, \cdots, k_{r}\right)}(a) \frac{t^{n}}{n !} \text {. }
$$

These numbers have the explicit formula involving Stirling numbers.

Theorem 1. For $k_{1}, k_{2}, \cdots, k_{r} \in \mathbb{Z}, n \geq 0$, we have:

$$
c_{n}^{\left(k_{1}, k_{2}, \cdots, k_{r}\right)}(a)=(-1)^{n} \sum_{0 \leq m_{1}<m_{2}<\cdots<m_{r}}^{n}\left(\frac{(-1)^{m_{r}}\left[\begin{array}{c}
n \\
m_{r}
\end{array}\right]}{\left(m_{r}+a\right)^{k_{r}}} \prod_{i=1}^{r-1} \frac{1}{m_{i} !\left(m_{i}+a-r+i\right)^{k_{i}}}\right) .
$$

Proof. First, we have:

$$
\begin{aligned}
\sum_{n=0}^{\infty} c_{n}^{\left(k_{1}, k_{2}, \cdots, k_{r}\right)}(a) \frac{t^{n}}{n !} & =\operatorname{\Phi f}\left(\ln (1+t),\left(k_{1}, k_{2}, \cdots, k_{r}\right), a\right) \\
& =\sum_{0 \leq m_{1}<m_{2}<\cdots<m_{r}} \frac{(\ln (1+t))^{m_{r}}}{m_{1} !\left(m_{1}+a-r+1\right)^{k_{1}} m_{2} !\left(m_{2}+a-r+2\right)^{k_{2}} \cdots m_{r} !\left(m_{r}+a\right)^{k_{r}}}
\end{aligned}
$$


Now, working on the right-hand side, we have:

$$
\begin{aligned}
& \sum_{0 \leq m_{1}<m_{2}<\cdots<m_{r}} \frac{(\ln (1+t))^{m_{r}}}{m_{1} !\left(m_{1}+a-r+1\right)^{k_{1}} m_{2} !\left(m_{2}+a-r+2\right)^{k_{2}} \cdots m_{r} !\left(m_{r}+a\right)^{k_{r}}} \\
& =\sum_{0 \leq m_{1}<m_{2}<\cdots<m_{r}}\left(\frac{(\ln (1+t))^{m_{r}}}{m_{r} !} \cdot \frac{1}{\left(m_{r}+a\right)^{k_{r}}} \prod_{i=1}^{r-1} \frac{1}{m_{i} !\left(m_{i}+a-r+i\right)^{k_{i}}}\right) \\
& =\sum_{0 \leq m_{1}<m_{2}<\cdots<m_{r}}\left(\sum_{n=m^{r}}^{\infty}(-1)^{n-m_{r}}\left[\begin{array}{c}
n \\
m_{r}
\end{array}\right] \frac{t^{n}}{n !} \cdot \frac{1}{\left(m_{r}+a\right)^{k_{r}}} \prod_{i=1}^{r-1} \frac{1}{m_{i} !\left(m_{i}+a-r+i\right)^{k_{i}}}\right) \\
& =\sum_{n=0}^{\infty} \sum_{0 \leq m_{1}<m_{2}<\cdots<m_{r}}^{n}\left(\frac{(-1)^{n-m_{r}}\left[\begin{array}{c}
n \\
m_{r}
\end{array}\right]}{\left(m_{r}+a\right)^{k_{r}}} \prod_{i=1}^{r-1} \frac{1}{m_{i} !\left(m_{i}+a-r+i\right)^{k_{i}}} \cdot \frac{t^{n}}{n !}\right) \\
& =\sum_{n=0}^{\infty}(-1)^{n} \sum_{0 \leq m_{1}<m_{2}<\cdots<m_{r}}^{n}\left(\frac{(-1)^{m_{r}}\left[\begin{array}{c}
n \\
m_{r}
\end{array}\right]}{\left(m_{r}+a\right)^{k_{r}}} \prod_{i=1}^{r-1} \frac{1}{m_{i} !\left(m_{i}+a-r+i\right)^{k_{i}}}\right) \frac{t^{n}}{n !} .
\end{aligned}
$$

It follows that:

$$
\sum_{n=0}^{\infty} c_{n}^{\left(k_{1}, k_{2}, \cdots, k_{r}\right)}(a) \frac{t^{n}}{n !}=\sum_{n=0}^{\infty}(-1)^{n} \sum_{0 \leq m_{1}<m_{2}<\cdots<m_{r}}^{n}\left(\frac{(-1)^{m_{r}}\left[\begin{array}{c}
n \\
m_{r}
\end{array}\right]}{\left(m_{r}+a\right)^{k_{r}}} \prod_{i=1}^{r-1} \frac{1}{m_{i} !\left(m_{i}+a-r+i\right)^{k_{i}}}\right) \frac{t^{n}}{n !} .
$$

Comparing the coefficients completes the proof.

The following theorem is an explicit formula for the Hurwitz-Lerch-type multi-poly-Cauchy numbers of the second kind, which can be shown similar to the proof of Theorem 1.

Theorem 2. For $k_{1}, k_{2}, \cdots, k_{r} \in \mathbb{Z}, n \geq 0$, we have:

$$
\hat{c}_{n}^{\left(k_{1}, k_{2}, \cdots, k_{r}\right)}(a)=(-1)^{n} \sum_{0 \leq m_{1}<m_{2}<\cdots<m_{r}}^{n}\left(\frac{\left[\begin{array}{c}
n \\
m_{r}
\end{array}\right]}{\left(m_{r}+a\right)^{k_{r}}} \prod_{i=1}^{r-1} \frac{1}{m_{i} !\left(m_{i}+a-r+i\right)^{k_{i}}}\right) .
$$

Parallel to the results of Cencki and Young [7], relations between Hurwitz-Lerch-type multi-poly-Cauchy numbers and Hurwitz-Lerch-type multi-poly-Bernoulli numbers can be shown using the orthogonality and inverse relations for Stirling numbers [12]. By the orthogonality relations:

$$
\sum_{r=m}^{n}(-1)^{n-r}\left[\begin{array}{l}
n \\
r
\end{array}\right]\left\{\begin{array}{c}
r \\
m
\end{array}\right\}=\sum_{r=m}^{n}(-1)^{n-r}\left\{\begin{array}{l}
n \\
r
\end{array}\right\}\left[\begin{array}{c}
r \\
m
\end{array}\right]=\delta_{m n}
$$

where $\delta_{m n}$ is a Kronecker symbol, it follows that:

$$
f_{n}=\sum_{m=0}^{n}(-1)^{n+m}\left[\begin{array}{c}
n \\
m
\end{array}\right] g_{m} \Longleftrightarrow g_{n}=\sum_{m=0}^{n}(-1)^{n+m}\left\{\begin{array}{c}
n \\
m
\end{array}\right\}
$$

Consequently, we obtain the following results:

Theorem 3. For the Hurwitz-Lerch-type multi-poly-Bernoulli numbers $B_{n}^{\left(k_{1}, k_{2}, \cdots, k_{r}\right)}(a)$, we have:

$$
\sum_{m_{r}=0}^{n}\left[\begin{array}{c}
n \\
m_{r}
\end{array}\right] B_{m_{r}}^{\left(k_{1}, k_{2}, \cdots, k_{r}\right)}(a)=\sum_{0 \leq m_{1} \leq \cdots \leq m_{r-1} \leq n}\left(\frac{n !}{(n+a)^{k_{r}}} \prod_{i=1}^{r-1} \frac{1}{\left(m_{i}+a-r+i\right)^{k_{i}}}\right) .
$$


Proof. Note that (3) can be expressed as:

$$
\begin{aligned}
B_{n}^{\left(k_{1}, k_{2}, \cdots, k_{r}\right)}(a) & =(-1)^{n} \sum_{0 \leq m_{1} \leq \cdots \leq m_{r} \leq n} \frac{(-1)^{m_{r}} m_{r} !\left\{\begin{array}{c}
n \\
m_{r}
\end{array}\right\}}{\left(m_{1}+a-r+1\right)^{k_{1}\left(m_{2}+a-r+2\right)^{k_{2}} \cdots\left(m_{r}+a\right)^{k_{r}}}} \\
& =\sum_{m_{r}=0}^{n} \sum_{0 \leq m_{1} \leq \cdots \leq m_{r}} \frac{(-1)^{n+m_{r}} m_{r} !\left\{\begin{array}{c}
n \\
m_{r}
\end{array}\right\}}{\left(m_{1}+a-r+1\right)^{k_{1}}\left(m_{2}+a-r+2\right)^{k_{2}} \cdots\left(m_{r}+a\right)^{k_{r}}} .
\end{aligned}
$$

Rewriting Equation (3) as:

$$
g_{n}=\sum_{m_{r}=0}^{n}\left\{\begin{array}{c}
n \\
m_{r}
\end{array}\right\} f_{m_{r}}
$$

for:

$$
g_{n}=(-1)^{n} B_{n}^{\left(k_{1}, k_{2}, \cdots, k_{r}\right)}(a)
$$

and:

$$
f_{m_{r}}=\sum_{0 \leq m_{1} \leq \cdots \leq m_{r}} \frac{(-1)^{m_{r}} m_{r} !}{\left(m_{1}+a-r+1\right)^{k_{1}\left(m_{2}+a-r+2\right)^{k_{2}} \cdots\left(m_{r}+a\right)^{k_{r}}},}
$$

the conclusion follows by applying Equation (10). so that:

Observe that when $r=1$, the right-hand side of Equation (11) reduces to the single term $\frac{n !}{(n+a)^{k_{1}}}$,

$$
\sum_{m_{1}=0}^{n}\left[\begin{array}{c}
n \\
m_{1}
\end{array}\right] B_{m_{1}}^{\left(k_{1}\right)}(a)=\frac{n !}{(n+a)^{k_{1}}}
$$

which is [7] (Theorem 2.5).

The next theorem contains result on Hurwitz-Lerch-type multi-poly-Cauchy numbers parallel to Theorem 3.

Theorem 4. For the Hurwitz-Lerch-type multi-poly-Cauchy numbers, we have:

$$
\sum_{m_{r}=0}^{n}\left\{\begin{array}{c}
n \\
m_{r}
\end{array}\right\} c_{m_{r}}^{\left(k_{1}, k_{2}, \cdots, k_{r}\right)}(a)=\sum_{0 \leq m_{1} \leq \cdots \leq m_{r-1} \leq n}\left(\frac{1}{(n+a)^{k_{r}}} \prod_{i=1}^{r-1} \frac{1}{m_{i} !\left(m_{i}+a-r+i\right)^{k_{i}}}\right)
$$

and:

$$
\sum_{m_{r}=0}^{n}\left\{\begin{array}{c}
n \\
m_{r}
\end{array}\right\} \hat{c}_{m_{r}}^{\left(k_{1}, k_{2}, \cdots, k_{r}\right)}(a)=\sum_{0 \leq m_{1} \leq \cdots \leq m_{r-1} \leq n}\left(\frac{(-1)^{n}}{(n+a)^{k_{r}}} \prod_{i=1}^{r-1} \frac{1}{m_{i} !\left(m_{i}+a-r+i\right)^{k_{i}}}\right) .
$$

Proof. The proof can be shown parallel to Theorem 3. Now, (12) follows from (7) and (10) by considering:

$$
f_{n}=c_{n}^{\left(k_{1}, k_{2}, \cdots, k_{r}\right)}(a)
$$

and:

$$
g_{m_{r}}=\sum_{0 \leq m_{1} \leq \cdots \leq m_{r}} \frac{1}{m_{1} !\left(m_{1}+a-r+1\right)^{k_{1}} \cdots m_{r-1} !\left(m_{r-1}+a-1\right)^{k_{r-1}\left(m_{r}+a\right)^{k_{r}}}},
$$

and (13) follows from (8) and (10) by considering:

$$
f_{n}=\hat{c}_{n}^{\left(k_{1}, k_{2}, \cdots, k_{r}\right)}(a)
$$


and:

$$
g_{m_{r}}=\sum_{0 \leq m_{1} \leq \cdots \leq m_{r}} \frac{(-1)^{m_{r}}}{m_{1} !\left(m_{1}+a-r+1\right)^{k_{1}} \cdots m_{r-1} !\left(m_{r-1}+a-1\right)^{k_{r-1}}\left(m_{r}+a\right)^{k_{r}}} .
$$

This completes the proof.

Now, when $r=1$, the right-hand side of (12) and (13) reduces to the single term $\frac{1}{(n+a)^{k_{1}}}$ and $\frac{(-1)^{n}}{(n+a)^{k_{1}}}$, respectively, so that:

$$
\sum_{m_{1}=0}^{n}\left\{\begin{array}{c}
n \\
m_{1}
\end{array}\right\} c_{m_{1}}^{\left(k_{1}\right)}(a)=\frac{1}{(n+a)^{k_{1}}}
$$

and:

$$
\sum_{m_{1}=0}^{n}\left\{\begin{array}{c}
n \\
m_{1}
\end{array}\right\} \hat{c}_{m_{1}}^{\left(k_{1}\right)}(a)=\frac{(-1)^{n}}{(n+a)^{k_{1}}}
$$

which is [7] (Theorem 2.6).

To obtain a kind of generalization of the results in [7] (Theorem 2.7), we introduce modified Hurwitz-Lerch-type multi-poly-Bernoulli numbers, denoted by $\hat{B}_{n}^{\left(k_{1}, k_{2}, \cdots, k_{r}\right)}(a)$, using the Hurwitz-Lerch multi-factorial zeta function as follows:

$$
\Phi f\left(1-e^{-t},\left(k_{1}, k_{2}, \cdots, k_{r}\right), a\right)=\sum_{n=0}^{\infty} \hat{B}_{n}^{\left(k_{1}, k_{2}, \cdots, k_{r}\right)}(a) \frac{t^{n}}{n !} .
$$

These numbers have the explicit formula involving Stirling numbers.

Lemma 1. For nonnegative integer $n$, we have:

$$
\hat{B}_{n}^{\left(k_{1}, k_{2}, \cdots, k_{r}\right)}(a)=\sum_{0 \leq m_{1}<m_{2} \cdots<m_{r}}^{n}\left(\frac{(-1)^{n+m_{r}}\left\{\begin{array}{c}
n \\
m_{r}
\end{array}\right\}}{(m+a)^{k_{r}}} \prod_{i=1}^{r-1} \frac{1}{m_{i} !\left(m_{i}+a-r+i\right)^{k_{i}}}\right) .
$$

Proof. Working on the left-hand side of (15), we get:

$$
\begin{aligned}
& \Phi f\left(1-e^{-t},\left(k_{1}, k_{2}, \cdots, k_{r}\right), a\right) \\
& =\sum_{0 \leq m_{1}<m_{2} \cdots<m_{r}}\left(\frac{\left(1-e^{-t}\right)^{m_{r}}}{m_{r} !(m+a)^{k_{r}}} \prod_{i=1}^{r-1} \frac{1}{m_{i} !\left(m_{i}+a-r+i\right)^{k_{i}}}\right) \\
& =\sum_{0 \leq m_{1}<m_{2} \cdots<m_{r}}\left(\frac{(-1)^{m_{r}}}{(m+a)^{k_{r}}} \frac{\left(e^{-t}-1\right)^{m_{r}}}{m_{r} !} \prod_{i=1}^{r-1} \frac{1}{m_{i} !\left(m_{i}+a-r+i\right)^{k_{i}}}\right) \\
& =\sum_{0 \leq m_{1}<m_{2} \cdots<m_{r}}\left(\frac{(-1)^{m_{r}}}{(m+a)^{k_{r}}} \sum_{n=m_{r}}\left\{\begin{array}{c}
n \\
m_{r}
\end{array}\right\} \frac{(-t)^{n}}{n !} \prod_{i=1}^{r-1} \frac{1}{m_{i} !\left(m_{i}+a-r+i\right)^{k_{i}}}\right) \\
& =\sum_{0 \leq m_{1}<m_{2} \cdots<m_{r}}\left(\frac{(-1)^{n+m_{r}}}{(m+a)^{k_{r}}} \sum_{n=m_{r}}\left\{\begin{array}{c}
n \\
m_{r}
\end{array}\right\} \frac{t^{n}}{n !} \prod_{i=1}^{r-1} \frac{1}{m_{i} !\left(m_{i}+a-r+i\right)^{k_{i}}}\right) \\
& =\sum_{n=0}^{\infty} \sum_{0 \leq m_{1}<m_{2} \cdots<m_{r}}^{n}\left(\frac{(-1)^{n+m_{r}}\left\{\begin{array}{c}
n \\
m_{r}
\end{array}\right\}}{(m+a)^{k_{r}}} \prod_{i=1}^{r-1} \frac{1}{m_{i} !\left(m_{i}+a-r+i\right)^{k_{i}}}\right) \frac{t^{n}}{n !} .
\end{aligned}
$$

Comparing the coefficients completes the proof. 
The next lemma is a result on the modified Hurwitz-Lerch-type multi-poly-Bernoulli numbers parallel to Theorem 3.

Lemma 2. For the Hurwitz-Lerch-type multi-poly-Bernoulli numbers, we have:

$$
\sum_{m_{r}=0}^{n}\left[\begin{array}{c}
n \\
m_{r}
\end{array}\right] \hat{B}_{m_{r}}^{\left(k_{1}, k_{2}, \cdots, k_{r}\right)}(a)=\sum_{0 \leq m_{1} \leq \cdots \leq m_{r-1} \leq n}\left(\frac{1}{(n+a)^{k_{r}}} \prod_{i=1}^{r-1} \frac{1}{m_{i} !\left(m_{i}+a-r+i\right)^{k_{i}}}\right) .
$$

Proof. Rewriting Lemma 1 as:

$$
g_{n}=\sum_{m_{r}=0}^{n}\left\{\begin{array}{c}
n \\
m_{r}
\end{array}\right\} f_{m_{r}}
$$

for:

$$
g_{n}=(-1)^{n} B_{n}^{\left(k_{1}, k_{2}, \cdots, k_{r}\right)}(a)
$$

and:

$$
f_{m_{r}}=\sum_{0 \leq m_{1} \leq \cdots \leq m_{r}}\left(\frac{(-1)^{m_{r}}}{\left(m_{r}+a\right)^{k_{r}}} \prod_{i=1}^{r-1} \frac{1}{m_{i} !\left(m_{i}+a-r+i\right)^{k_{i}}}\right),
$$

and the conclusion follows by applying (7).

The next theorem contains the desired relationship between the Hurwitz-Lerch-type multi-poly-Cauchy numbers and the modified Hurwitz-Lerch-type multi-poly-Bernoulli numbers.

Theorem 5. For nonnegative integer $n$, we have:

$$
\begin{aligned}
& \hat{B}_{n}^{\left(k_{1}, k_{2}, \cdots, k_{r}\right)}(a)=\sum_{l_{r}=0}^{n} \sum_{m_{r}=0}^{n}(-1)^{m_{r}+n}\left\{\begin{array}{c}
n \\
m_{r}
\end{array}\right\}\left\{\begin{array}{c}
m_{r} \\
l_{r}
\end{array}\right\} c_{l_{r}}^{\left(k_{1}, k_{2}, \cdots, k_{r}\right)}(a), \\
& \hat{B}_{n}^{\left(k_{1}, k_{2}, \cdots, k_{r}\right)}(a)=\sum_{l_{r}=0}^{n} \sum_{m_{r}=0}^{n}(-1)^{m_{r}}\left\{\begin{array}{c}
n \\
m_{r}
\end{array}\right\}\left\{\begin{array}{c}
m_{r} \\
l_{r}
\end{array}\right\} \hat{c}_{l_{r}}^{\left(k_{1}, k_{2}, \cdots, k_{r}\right)}(a), \\
& c_{n}^{\left(k_{1}, k_{2}, \cdots, k_{r}\right)}(a)=\sum_{l_{r}=0}^{n} \sum_{m_{r}=0}^{n}(-1)^{m_{r}+n}\left[\begin{array}{c}
n \\
m_{r}
\end{array}\right]\left[\begin{array}{c}
m_{r} \\
l_{r}
\end{array}\right] \hat{B}_{l_{r}}^{\left(k_{1}, k_{2}, \cdots, k_{r}\right)}(a), \\
& \hat{c}_{n}^{\left(k_{1}, k_{2}, \cdots, k_{r}\right)}(a)=\sum_{l_{r}=0}^{n} \sum_{m_{r}=0}^{n}(-1)^{n}\left[\begin{array}{c}
n \\
m_{r}
\end{array}\right]\left[\begin{array}{c}
m_{r} \\
l_{r}
\end{array}\right] \hat{B}_{l_{r}}^{\left(k_{1}, k_{2}, \cdots, k_{r}\right)}(a) .
\end{aligned}
$$


Proof. Using Lemma 1 and (12), we have:

$$
\begin{aligned}
& \sum_{l_{r}=0}^{n} \sum_{m_{r}=0}^{n}(-1)^{m_{r}+n}\left\{\begin{array}{c}
n \\
m_{r}
\end{array}\right\}\left\{\begin{array}{c}
m_{r} \\
l_{r}
\end{array}\right\} c_{l_{r}}^{\left(k_{1}, k_{2}, \cdots, k_{r}\right)}(a) \\
= & \sum_{l_{r}=0}^{n} \sum_{m_{r}=l_{r}}^{n}(-1)^{m_{r}+n}\left\{\begin{array}{c}
n \\
m_{r}
\end{array}\right\}\left\{\begin{array}{c}
m_{r} \\
l_{r}
\end{array}\right\} c_{l_{r}}^{\left(k_{1}, k_{2}, \cdots, k_{r}\right)}(a) \\
= & \sum_{m_{r}=0}^{n}(-1)^{m_{r}+n}\left\{\begin{array}{c}
n \\
m_{r}
\end{array}\right\} \sum_{l_{r}=0}^{m_{r}}\left\{\begin{array}{c}
m_{r} \\
l_{r}
\end{array}\right\} c_{l_{r}}^{\left(k_{1}, k_{2}, \cdots, k_{r}\right)}(a) \\
= & \sum_{m_{r}=0}^{n}(-1)^{m_{r}+n}\left\{\begin{array}{c}
n \\
m_{r}
\end{array}\right\} \sum_{0 \leq l_{1} \leq \cdots \leq l_{r-1} \leq m_{r}}\left(\frac{1}{\left(m_{r}+a\right)^{k_{r}}} \prod_{i=1}^{r-1} \frac{1}{l_{i} !\left(l_{i}+a-r+i\right)^{k_{i}}}\right) \\
= & \sum_{0 \leq l_{1} \leq \cdots \leq l_{r-1} \leq m_{r}}^{n}\left(\frac{(-1)^{m_{r}+n}\left\{\begin{array}{c}
n \\
\left.m_{r}\right\}
\end{array} \prod^{r-1} \frac{1}{\left(m_{r}+a\right)^{k_{r}}} \prod_{i=1}^{r !\left(l_{i}+a-r+i\right)^{k_{i}}}\right)}{=} \hat{B}_{n}^{\left(k_{1}, k_{2}, \cdots, k_{r}\right)}(a) .\right.
\end{aligned}
$$

Now, (17) can be found using Lemma 1 and (13). For (18), using (7) and Lemma 2 yields:

$$
\begin{aligned}
& \sum_{l_{r}=0}^{n} \sum_{m_{r}=0}^{n}(-1)^{m_{r}+n}\left[\begin{array}{c}
n \\
m_{r}
\end{array}\right]\left[\begin{array}{c}
m_{r} \\
l_{r}
\end{array}\right] \hat{B}_{l_{r}}^{\left(k_{1}, k_{2}, \cdots, k_{r}\right)}(a) \\
= & \sum_{l_{r}=0}^{n} \sum_{m_{r}=l_{r}}^{n}(-1)^{m_{r}+n}\left[\begin{array}{c}
n \\
m_{r}
\end{array}\right]\left[\begin{array}{c}
m_{r} \\
l_{r}
\end{array}\right] \hat{B}_{l_{r}}^{\left(k_{1}, k_{2}, \cdots, k_{r}\right)}(a) \\
= & \sum_{m_{r}=0}^{n}(-1)^{m_{r}+n}\left[\begin{array}{c}
n \\
m_{r}
\end{array}\right] \sum_{l_{r}=0}^{m_{r}}\left[\begin{array}{c}
m_{r} \\
l_{r}
\end{array}\right] \hat{B}_{l_{r}}^{\left(k_{1}, k_{2}, \cdots, k_{r}\right)}(a) \\
= & \sum_{m_{r}=0}^{n}(-1)^{m_{r}+n}\left[\begin{array}{c}
n \\
m_{r}
\end{array}\right] \sum_{0 \leq l_{1} \leq \cdots \leq l_{r-1} \leq m_{r}}\left(\frac{1}{\left(m_{r}+a\right)^{k_{r}}} \prod_{i=1}^{r-1} \frac{1}{l_{i} !\left(l_{i}+a-r+i\right)^{k_{i}}}\right) \\
= & \sum_{0 \leq l_{1} \leq \cdots \leq l_{r-1} \leq m_{r}}^{n}\left(\frac{(-1)^{m_{r}+n}\left[\begin{array}{l}
n \\
m_{r}
\end{array}\right]}{\left(m_{r}+a\right)^{k_{r}}} \prod_{i=1}^{r-1} \frac{1}{l_{i} !\left(l_{i}+a-r+i\right)^{k_{i}}}\right) \\
= & \hat{c}_{n}^{\left(k_{1}, k_{2}, \cdots, k_{r}\right)}(a) .
\end{aligned}
$$

Similarly, (19) can be obtained using (8) and Lemma 2.

When $r=1$, (16), (17), (18), and (19) yield:

$$
\begin{aligned}
& \hat{B}_{n}^{(k)}(a)=\sum_{l=0}^{n} \sum_{m=0}^{n}(-1)^{m+n}\left\{\begin{array}{c}
n \\
m
\end{array}\right\}\left\{\begin{array}{c}
m \\
l
\end{array}\right\} c_{l}^{(k)}(a), \\
& \hat{B}_{n}^{(k)}(a)=\sum_{l=0}^{n} \sum_{m=0}^{n}(-1)^{m}\left\{\begin{array}{c}
n \\
m
\end{array}\right\}\left\{\begin{array}{c}
m \\
l
\end{array}\right\} \hat{c}_{l}^{(k)}(a), \\
& c_{n}^{(k)}(a)=\sum_{l=0}^{n} \sum_{m=0}^{n}(-1)^{m+n}\left[\begin{array}{c}
n \\
m
\end{array}\right]\left[\begin{array}{c}
m \\
l
\end{array}\right] \hat{B}_{l}^{(k)}(a), \\
& \hat{c}_{n}^{(k)}(a)=\sum_{l=0}^{n} \sum_{m=0}^{n}(-1)^{n}\left[\begin{array}{c}
n \\
m
\end{array}\right]\left[\begin{array}{c}
m \\
l
\end{array}\right] \hat{B}_{l}^{(k)}(a) .
\end{aligned}
$$

where $\hat{B}_{n}^{(k)}(a)$ is the modified Hurwitz-Lerch-type poly-Bernoulli numbers defined by:

$$
\Phi f\left(1-e^{-t}, k, a\right)=\sum_{n=0}^{\infty} \hat{B}_{n}^{(k)}(a) \frac{t^{n}}{n !} .
$$


Author Contributions: The authors contributed equally to this work.

Funding: This research received no external funding.

Acknowledgments: The authors would like to express their sincere gratitude to the Editor and referees for their corrections and improvement of this paper.

Conflicts of Interest: The authors declare no conflict of interest.

\section{References}

1. Comtet, L. Advanced Combinatorics. The Art of Finite and Infinite Expansions; D. Reidel Publishing Company: Dordrecht, The Netherland, 1974; pp. 293-294.

2. Komatsu, T. Poly-Cauchy numbers. Kyushu J. Math. 2013, 67, 143-153. [CrossRef]

3. Merlini, D.; Sprugnoli, R.; Verri, M.C. The Cauchy Numbers. Discrete Math. 2006, 306, 1906-1920. [CrossRef]

4. Knuth, D. Two notes on notation. Am. Math. Monthly 1992, 99, 403-422. [CrossRef]

5. Kaneko, M. Poly-Bernoulli numbers. J. Théor. Nr. Bordx. 1997, 9, 221-228. [CrossRef]

6. Komatsu, T.; Laohakosol, V.; Liptai, K. A generalization of poly-Cauchy numbers and their properties. Abstr. Appl. Anal. 2013, 2013, 179841. [CrossRef]

7. Cenkci, M.; Young, P.T. Generalizations of poly-Bernoulli and poly-Cauchy numbers. Eur. J. Math. 2015, 1, 799-828. [CrossRef]

8. Corcino, R.B.; Jolany, H.; Corcino, C.B.; Komatsu, T. On Multi Poly-Bernoulli Polynomials. Available online: https:/ / arxiv.org/abs/1607.03746 (accessed on 23 March 2018).

9. Corcino, R.B. Multi Poly-Bernoulli and Multi Poly-Euler Polynomials. In Applied Mathematical Analysis: Theory, Methods and Applications; Dutta, H., Peters, J., Eds.; Studies in Systems, Decision and Control 177; Springer: Cham, Switzerland, 2020; pp. 679-721. [CrossRef]

10. Kim, D.S.; Kim, T.; Komatsu, T.; Rim, S.-H. Multi-Poly-Cauchy polynomials. J. Comput. Anal. Appl. 2015, 18, 536-550.

11. Imatomi, K.; Kaneko, M.; Tekada, E. Multi-poly-Bernoulli Numbers and Finite Multiple Zeta Values. J. Integer Seq. 2014, 17, 14.4.5.

12. Broder, A.Z. The r-Stirling numbers. Discrete Math. 1984, 49, 241-259. [CrossRef]

(C) 2019 by the authors. Licensee MDPI, Basel, Switzerland. This article is an open access article distributed under the terms and conditions of the Creative Commons Attribution (CC BY) license (http://creativecommons.org/licenses/by/4.0/). 